\title{
Spindle Coma in the Intensive Care Unit: Different Aetiologies - Different Outcomes
}

\author{
Ana Catarina Emidio ${ }^{1}$, Rita Faria², Patricia Patricio ${ }^{2}$, Nuno Canas ${ }^{3}$, António Messias ${ }^{2}$, Carlos Meneses-Oliveira² \\ ${ }^{1}$ Internal Medicine Department, Hospital de São Bernardo, Centro Hospitalar de Setúbal, Portugal \\ ${ }^{2}$ Intensive Medicine Department, Hospital Beatriz Ângelo, Loures, Portugal \\ ${ }^{3}$ Neurophysiology laboratory, Neurology Department, Hospital Beatriz Ângelo, Loures, Portugal
}

\section{Doi: 10.12890/2019_001316 - European Journal of Case Reports in Internal Medicine - ๑ EFIM 2019}

Received: 07/10/2019

Accepted: $15 / 10 / 2019$

Published: 15/11/2019

\begin{abstract}
How to cite this article: Emidio AC, Faria R, Patricio P, Canas N, Messias A, Meneses-Oliveira C. Spindle coma in the intensive care uniti: different
\end{abstract} aetiologies- different outcomes. EJCRIM 2019;6: doi:10.12890/2019_001316.

Conflicts of Interests: The Authors declare that there are no competing interest

This article is licensed under a Commons Attribution Non-Commercial 4.0 License

\section{ABSTRACT}

Spindle coma is an electroclinical entity that has been used to describe an EEG pattern of "sleep-like" activity in comatose patients. Although it has been associated with favourable prognosis, its aetiology is one of the key factors for patient outcome. The authors present three cases of spindle coma with different aetiologies (amitriptyline overdose, pontine myelinolysis and hypoxic-ischaemic encephalopathy) that culminated in different outcomes.

\section{LEARNING POINTS}

- Spindle coma (SC) is an electroclinical "sleep-like" pattern that appears in comatose patients.

- It implies the functional preservation of the cerebral hemispheres which can be associated with a good prognosis.

- The aetiology of SC is a key factor for outcome.

\section{KEYWORDS}

Spindle coma, coma, EEG, physiopathology

\section{INTRODUCTION}

The reversed halo sign (RHS) was initially considered to be a specific sign of organising pneumonia (OP). However, it has been described in a variety of pulmonary diseases. We report a case of an unexpected diagnosis of pulmonary tuberculosis (PT) that was initially thought to be OP due to the presence of this sign, reinforcing the importance of excluding other possible aetiologies when the RHS is present.

\section{CASE PRESENTATION}

\section{Case 1}

A 71-year-old woman with an amitriptyline overdose (approximately $850 \mathrm{mg}-12 \mathrm{mg} / \mathrm{kg}$ ) was admitted to the intensive care unit (ICU). She was in a coma with a Glasgow Coma Score (GCS) of 3 and required intubation for airway protection. An electrocardiogram revealed a prolonged QTc (490 ms) and blood analysis showed tricyclic antidepressant dosage higher than 2,510 mcg/dl while there were no metabolic or electrolytical disturbances. The brain computed tomography (CT)-angiography did not show any lesions. The electroencephalogram (EEG) performed on the first day of hospitalisation (Fig. 1a) showed a monomorphic continuous pattern of sleep spindles with no reactivity to external stimuli (Fig. 1b). During drug wash-out, the patient displayed progressively improved consciousness. On the 4th day of hospitalisation, she had a GCS of 14, and was discharged to the ward on the 8th day with a GCS of 15. 


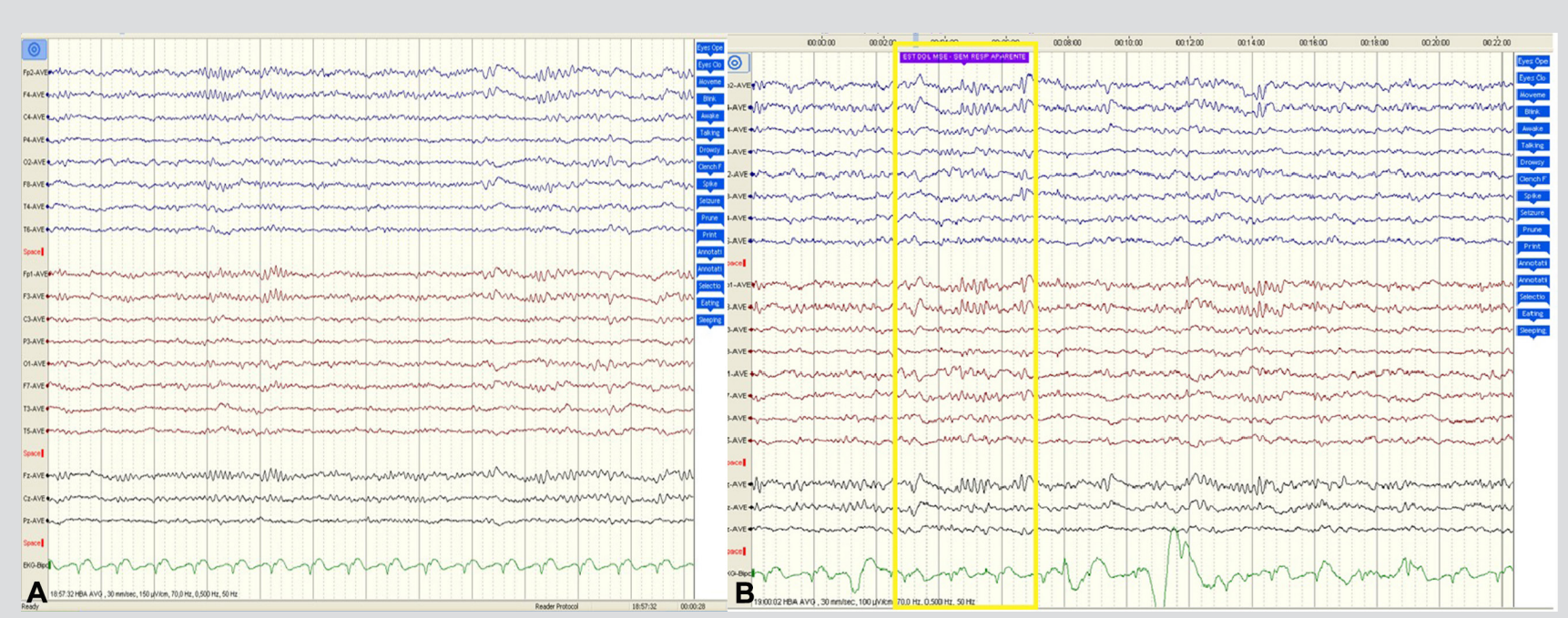

Figure 1. EEG showing sleep-like pattern (a) with no response to external stimuli (yellow rectangle) (b)

Case 2

A 50-year-old diabetic woman was transferred from a hospital in Angola, where she was admitted with nausea, vomiting and epigastralgia. Laboratory evaluation less than 36 hours before the admission to our emergency department was significant for a sodium level of 150 $\mathrm{mEq} / \mathrm{l}$. On admission, the patient had a sodium level of $177 \mathrm{mEq} / \mathrm{l}$ and blood glucose of $178 \mathrm{mg} / \mathrm{dl}$. After 12 hours of intravenous fluid therapy ( $\mathrm{NaCl}$ 0.45\%) and insulin, the patient became confused with altered mental status (GCS 9). She was admitted to the ICU and intubated for airway protection. Laboratory re-evaluation showed a sodium level of $160 \mathrm{mmol} / \mathrm{l}$. After sedation interruption, she persisted in a coma (GCS 3T) with a flaccid tetraparesis. The EEG showed a sleep spindle pattern without response to external stimuli. The brain magnetic resonance imaging (MRI) scan (Fig. 2) was compatible with pontine myelinolysis associated with the elevation of blood sodium levels. Other causes of SC (trauma, drugs, neoplasm or other metabolic disturbances) were excluded based on history, clinical examination and radiological and laboratory investigations. The patient was discharged to the ward on the 22nd day of admission, with a GCS of 15 and maintaining flaccid tetraparesis. After 3 months of rehabilitation, the patient persists with tetraparesis MRC grade 4/5 in the lower limbs and MRC grade $3 / 5$ in the upper limbs.

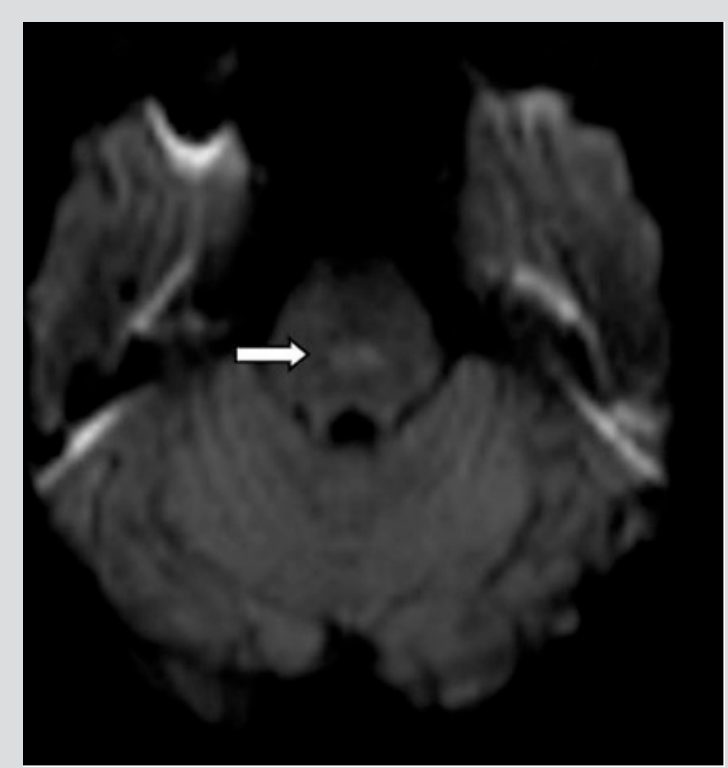

Figure 2. Brain MRI scan compatible with pontine myelinolysis (arrow) 
Case 3

A 55-year-old woman was admitted to the ICU with septic shock (resulting from osteomyelitis after L4-L5 arthrodesis). She required intubation after respiratory failure. Despite directed antibiotic therapy and surgical debridement, source control was not achieved. She maintained an altered state of consciousness with a fluctuating GCS (5T-11T); the brain CT scan was without acute lesions and the EEG suggested metabolic encephalopathy. During hospitalisation, numerous hypoxic-ischaemic insults occurred, including a prolonged period of hypoxaemia associated with cardio-respiratory arrest. On the 27th day of hospitalisation, the patient persisted in a coma and the EEG disclosed a sleep spindle pattern with no reactivity to external stimuli. The brain MRI scan showed an ischaemic lesion in the paramedian pons. The patient died on the 61st day of hospitalisation, despite all efforts to treat her.

\section{DISCUSSION}

SC was first described in 1953 in patients with hypothalamic neoplasms and since then it has been associated with traumatic and nontraumatic aetiologies ${ }^{[1]}$. It is an electroclinical entity that has been used to describe an EEG pattern of "sleep-like" activity characterised by spindles, vertex waves and K-complexes in patients with unconsciousness or coma. Although the background is predominantly theta and delta activity, frequent 11 to $14 \mathrm{~Hz}$ spindle discharges are often present in a symmetric and synchronous manner ${ }^{[1]}$. The pathophysiological mechanism of SC is presumed to be the preservation of pontine raphe nuclei and thalamocortical circuits subserving sleep spindle activity, together with the impairment of ascending reticular activating pathways at the midbrain level that maintain wakefulness ${ }^{[2]}$.

The demonstration of SC in the EEG is generally associated with a good prognosis for clinical recovery, since it suggests functional preservation of both cerebral hemispheres ${ }^{[1,3]}$. However, at the present time, the role of SC as a diagnostic or prognostic marker is controversial, depending on numerous facts: age, aetiology, depth of coma, duration of the lesion, EEG reactivity to external stimuli and the presence of brain stem reflexes ${ }^{[1,3,4]}$.

Like other EEG patterns, SC is not specific for a single aetiology, being associated with several causes: trauma, cerebrovascular disease, neoplasms, surgery, anoxic encephalopathy, seizures, metabolic disturbances or drug intoxications ${ }^{[1,3]}$. Among drug intoxications, the most frequent are benzodiazepines and barbiturates, with only one case describing SC with tricyclic antidepressant overdose ${ }^{[5]}$, like that which occurred in our first case. The prognosis in cases of drug intoxications is favourable with adequate treatment, with SC reactivity having less value for outcome prediction ${ }^{[4]}$.

In our second case, the patient had a metabolic disturbance conditioning the occurrence of impaired mental status: hypernatraemia caused by the fast augmentation of sodium ( $27 \mathrm{mEq} / \mathrm{l}$ in less than 36 hours). The EEG pattern is highly variable in metabolic disturbances and SC can also occur ${ }^{[3]}$. In this case, although there was an absence of SC reactivity to external stimuli, the patient recovered consciousness and the prognosis was defined by the underlying cause - pontine myelinolysis.

The third case illustrates the development of SC as a consequence of a prolonged period of hypoxia. As in the previous cases, there was a lack of SC reactivity to external stimuli. In fact, the occurrence of spindle pattern both in patients with cerebrovascular disease or after hypoxia is associated with a better prognosis when compared to the prognosis of those without this pattern ${ }^{[3]}$. However, the severity of the septic shock, the prolonged period of mechanical ventilation and the ICU stay were decisive for the outcome.

With these cases, the authors want to emphasise that although SC, and namely, its reactivity to external stimuli, has been associated with a good prognosis, this depends mainly on the coma aetiology. In this way, when confronted with a patient with SC, clinicians should consider a broad differential diagnosis and reserve judgment about the prognosis until an underlying cause is identified.

\section{REFERENCES}

1. Husain A. Electroencephalographic assessment of coma. J Clin Neurophysiol 2006;23:208-220.

2. Cologana V, Schabusa M, Ledouxc D, Moonend G, Maqueta P, Laureysa S. Sleep in disorders of consciousness. Sleep Med Rev 2010;14:97-105.

3. Kaplan P, Genoud D, Ho T, Jallon P. Clinical correlates and prognosis in early spindle coma. Clin Neurophysiol 2000;111:584-590.

4. Brenner R. The interpretation of the EEG in stupor and coma. Neurologist 2005;11:271-284.

5. Pulst S, Lombroso C. External ophthalmoplegia, alpha and spindle coma in imipramine overdose: case report and review of the literature. Ann Neurol 1983;14:587-590. 